\title{
Chandra follow-up of bursters with low persistent emission
}

\author{
R. Cornelisse ${ }^{1,2}$, F. Verbunt ${ }^{2}$, J. J. M. in 't Zand ${ }^{2,1}$, E. Kuulkers ${ }^{1,2, \star}$, and J. Heise ${ }^{1}$ \\ 1 SRON National Institute for Space Research, Sorbonnelaan 2, 3584 CA Utrecht, The Netherlands \\ 2 Astronomical Institute, Utrecht University, PO Box 80000, 3508 TA Utrecht, The Netherlands
}

Received 13 February 2002 / Accepted 21 June 2002

\begin{abstract}
We report on Chandra ACIS-S observations of five type I X-ray bursters with low persistent emission: SAXJ1324.5-6313, SAXJ1752.3-3128, SAXJ1753.5-2349, SAXJ1806.5-2215, and SAXJ1818.7+1424. We designate candidate persistent sources for four X-ray bursters. All candidates are detected at a persistent luminosity level of $10^{32-33} \mathrm{erg} \mathrm{s}^{-1}$, comparable to soft X-ray transients in quiescence. From the number of bursters with low persistent emission detected so far with the Wide Field Cameras, we estimate a total of such sources in our Galaxy between 30 and 4000.
\end{abstract}

Key words. binaries: close - stars: individual: SAX J1324.5-6313, SAX J1752.3-3128, SAX J1753.5-2349,

SAX J1806.5-2215, SAX J1818.7+1424 - stars: neutron - X-rays: bursts

\section{Introduction}

Many low-mass X-ray binaries show bursts of X-rays which are characterized by a rapid rise and exponential decay, and by a black body spectrum with spectral softening during the decay i.e. the emitter cools. Such type I X-ray bursts are interpreted as thermonuclear flashes on surfaces of neutron stars, and thus effectively identify the emitting source as a neutron star as opposed to a black hole. The theory of these bursts predicts a relation between the accretion rate onto the neutron star, as measured by the persistent X-ray luminosity, and the properties of the X-ray burst. Briefly, for very low and very high accretion rates, no X-ray bursts are expected, because thermonuclear fusion is steady (Fujimoto et al. 1987). At intermediate accretion rates, hydrogen/helium fusion occurs sporadically in bursts, and the burst frequency is a function of the accretion rate per square meter on the neutron star. Because the effectively accreting area of the neutron star is also a function of the accretion rate, the burst frequency is a non-monotonic function of the persistent X-ray luminosity. Recent reviews of burst theory are given by Bildsten (1998, 2000).

Low-mass X-ray binaries are discovered as either persistent sources or transient sources. The transient sources with neutron stars show outbursts lasting for weeks, sometimes up to years, at luminosities above $10^{36} \mathrm{erg} \mathrm{s}^{-1}$. During their quiescent state their luminosity drops to a level of $10^{32-33} \mathrm{erg} \mathrm{s}^{-1}$ (e.g. Campana et al. 1998), and the time averaged luminosities are $\lesssim 10^{36} \mathrm{erg} \mathrm{s}^{-1}$ (e.g. White et al. 1984). Most bursts are emitted by systems at luminosities $\gtrsim 10^{36} \mathrm{erg} \mathrm{s}^{-1}$, e.g. the transients

Send offprint requests to: $\mathrm{R}$. Cornelisse,

e-mail: R.Cornelisse@sron.nl

* Present address: ESA-ESTEC, Science Operations and Data Systems Division, SCI-SDG, Keplerlaan 1, 2201 AZ Noordwijk, The Netherlands.
Aq1 X-1 and Cen X-4 emitted X-ray bursts when they were in outburst (Koyama et al. 1981; Matsuoka et al. 1980).

The Wide Field Cameras (WFC) on board the Italian-Dutch Satellite BeppoSAX discovered sporadic type I bursts from nine previously unknown burst sources, which had persistent X-ray fluxes below the WFC detection limit of a few times $10^{-10} \mathrm{erg} \mathrm{cm}^{-2} \mathrm{~s}^{-1}(2-28 \mathrm{keV})$. At $8 \mathrm{kpc}$, the distance of the Galactic center, these flux limits correspond to luminosities of $\sim 10^{36} \mathrm{erg} \mathrm{s}^{-1}$. Four of the nine previously unknown burst sources were detected with other instruments at fluxes well below the WFC detection limit (see Table 1). The five other bursters are listed in Table 2. In this article we present Chandra observations which we obtained in order to determine the flux levels of these five burst sources.

The persistent luminosities of the nine previously unknown burst sources are (possibly far) below $10^{36} \mathrm{erg} \mathrm{s}^{-1}$, i.e. below the level X-ray bursts are usually observed. This is the reason why Cocchi et al. (2001) suggested that these sources are members of a new class of bursters with low persistent emission (see also Cornelisse et al. 2002).

The nine sources can be used to explore the low end of the relation between luminosity and burst properties. The long waiting times between type I bursts, compared to brighter burst sources, plus the low persistent emission level make these sources difficult to discover. Its large field of view makes the WFC an efficient instrument for the detection of such rare events.

In Sect. 2 we describe the Chandra observations and data analysis and in Sect. 3 we discuss which of the detected sources are the most likely candidates for each burster. In Sect. 4 we briefly present unpublished but relevant observations with other instruments of SAXJ1806.5-2215 and GRS 1741.9-2853. 
Table 1. Overview of the detection of four of the low persistent emission bursters. For each source we list the instrument which detected the source, the date of observation and the persistent flux in $10^{-11} \mathrm{erg} \mathrm{cm}^{-2} \mathrm{~s}^{-1}$ plus passband in $\mathrm{keV}$. References: a. Kaptein et al. (2000), b. Cocchi et al. (1999), c. Pavlinsky et al. (1994), d. Cornelisse et al. (2002), f. Antonelli et al. (1999), g. in 't Zand et al. (2002, in preparation).

\begin{tabular}{lccccc}
\hline \hline source & instrument & date & $F$ & range & ref. \\
\hline 1RXS J1718.4-4029 & ROSAT/P & 1990 & 1 & $2-10$ & $\mathrm{a}$ \\
1RXS J1718.4-4029 & ROSAT/H & 1994 & 0.4 & $2-10$ & $\mathrm{a}$ \\
GRS 1741.9-2853 & GRANAT & 1990 & 19 & $4-30$ & $\mathrm{~b}, \mathrm{c}$ \\
SAX J1828.5-1037 & ROSAT/P & 1993 & 0.19 & $0.5-2.5$ & $\mathrm{~d}$ \\
SAX J2224.9+5421 $^{e}$ & SAX/NFI & 1999 & 0.013 & $2-10$ & f, g \\
\hline
\end{tabular}

${ }^{e}$ Observation a few hours after burst.

In Sect. 5 we discuss the implications for the class of low persistent emission bursters.

\section{Observations and data analysis}

With the Chandra satellite (Weisskopf 1988) we observed the WFC error circles of the five burst sources without persistent emission listed in Table 2. For each field we used the ACIS-S3 detector in imaging mode. We analyzed the level 2 FITS data provided with the standard data products using the Chandra Interactive Analysis of Observations Software (CIAO) version 2.1.3. None of the five observations showed periods of high background and we used all data. For source detection we used a wavelet-based algorithm (Freeman et al. 2002), only taking into account the events between 0.5 and $7 \mathrm{keV}$. We set the significance threshold for the source detection at $10^{-6}$, i.e. giving at most one spurious source on the ACIS-S3 detector per observation. In the dithered detector image we checked each source region for the presence of flickering pixels. If a pixel detected more than one photon from a source during the whole observation we marked this as a flickering pixel; given the small number of photons in each source (see Table 3 ) we think that the chance probability that this happens is too small $\left(\simeq 10^{-5}\right)$ to be coincidence. In Table 3 we have noted the source which is affected by a flickering pixel with an $\mathrm{f}$. The count rate and position are not reliable for this source. The wavelet method also gives an estimate of the background. We consider all sources detected with a significance of more than $3 \sigma$. Only for the observation of SAX J1753.5-2349 no sources above $3 \sigma$ were detected; here we derive an upper limit of 5 counts.

\section{Selecting candidate burst sources}

In Table 3 we list the detected sources on the whole S3-chip for each observation, because there is still a $1 \%$ possibility that the source is outside the error circle. In all four observations there is more than one source inside or close to the WFC error circles. Based on the photon count rate, there are no extreme examples of sources which would qualify them as particularly likely candidates. This could very well mean that all detected sources are spurious and none are the bursters, also given that no source was detected during the observation of SAX J1753.5-2349.
Table 2. Observation log of the bursters at low persistent emission. For each source we list the start and exposure time of the Chandra observation, the WFC error radius ( $99 \%$ confidence), the absorption column $\left(N_{\mathrm{H}}\right.$, in $10^{21}$ atoms cm $\left.\mathrm{cm}^{-2}\right)$ as found by interpolating the $\mathrm{HI}$ maps of Dickey \& Lockman (1990), and the upper limit to the distance $\left(d_{\mathrm{u}}\right.$, in kpc) derived from the burst peak flux. For comparison with a model spectrum of a neutron star $\mathrm{H}$-atmosphere plus powerlaw we also list for this model the absorbed flux $(F, 0.5-7 \mathrm{keV}$, in $10^{-12} \mathrm{erg} \mathrm{cm}^{-2} \mathrm{~s}^{-1}$ ) corresponding to 1 Chandra count per second, and the absorbed softness-ratio (SR) of the soft count rate $(0.5-2 \mathrm{keV})$ to the total count rate $(0.5-7 \mathrm{keV})$. References: a. Cornelisse et al. (2002), b. Cocchi et al. (2001), c. in 't Zand et al. (1998), d. this paper.

\begin{tabular}{cccccccccc}
\hline \hline $\begin{array}{c}\text { source } \\
(\text { SAX })\end{array}$ & $\begin{array}{c}\text { start date } \\
(\mathrm{MJD})\end{array}$ & $\begin{array}{c}\text { exp. } \\
(\mathrm{s})\end{array}$ & $\begin{array}{c}\delta \\
\left({ }^{\prime}\right)\end{array}$ & $N_{\mathrm{H}}$ & $d_{\mathrm{u}}$ & $F$ & SR & ref. \\
\hline $1324.5-6313$ & 52162.39 & 5101 & 1.8 & 15 & 6.2 & 1.0 & 0.69 & $\mathrm{a}$ \\
$1752.3-3128$ & 52174.20 & 4717 & 2.9 & 5.6 & 9.2 & 2.5 & 0.82 & $\mathrm{~b}$ \\
$1753.5-2349$ & 52187.83 & 5171 & 2.5 & 8.3 & 8.8 & 1.6 & 0.76 & $\mathrm{c}$ \\
$1806.5-2215$ & 52206.39 & 4758 & 2.9 & 12 & 8.0 & 1.5 & 0.68 & $\mathrm{~d}$ \\
$1818.7+1424$ & 52092.17 & 4758 & 2.9 & 1.0 & 9.4 & 5.5 & 0.94 & $\mathrm{a}$ \\
\hline
\end{tabular}

Thus, we resort to several criteria to select viable burster candidates. We start with excluding all sources outside the WFC error circles. In Fig. 1 we see that several X-ray sources are close to optical sources from the Sloan Digitized Sky Survey. We have listed the closest star from the USNO catalogue within $4 "$ of the X-ray sources in Table 4. Given the small number of counts we estimate an error in the X-ray position of 1 pixel, i.e. 0.5, and a systematic error of another pixel. This gives a total error of $0{ }^{\prime} 7$. The positional error for the sources in the USNO Digitized Sky Survey is negligible in comparison. For each source in Table 4 we count the number of stars from the USNO catalogue inside the WFC error-circle and brighter than the potential counterpart. On the basis of this number we estimate the chance coincidence as in the following example. In the USNO catalogue the star closest to source D of SAX J1324.5-6313 is at 0.'83 (see Table 4). We find 24 stars in the USNO catalogue inside the WFC error circle of 1.8 that are brighter than $B=17.2$ and $R=15.3$. This gives a chance probability of $0.14 \%$ that one arbitrarily chosen position falls within one of the 24 error circles of 0. '83. The five Chandra sources inside the WFC error circle correspond to five trials, i.e. the chance probability that one or more of these sources are close to a star is $0.71 \%$. In Table 4 we have listed the chance probabilities $P$ thus computed for all Chandra sources within $4^{\prime \prime}$ of an optical star.

Although it is difficult to draw firm conclusions from a posteriori statistics, we think that the optical counterparts with $P<0.1 \%$ are secure. The optical sources located at a distance $\Delta \gtrsim 1^{\prime \prime}$ from the X-ray position are very likely chance coincidences. This leaves only D of the SAX J1324.5-6313 field as a borderline case, which may or may not be the counterpart. We have indicated the optically identified sources in Table 3 with an asterisk.

Assuming that the $V$ magnitudes are between the $B$ and $R$ magnitudes given in the USNO Digitized Sky Survey, we find that the X-ray to optical flux ratios of these stars are well within the range of the coronal emission from normal nearby 
Table 3. For each source detected on the S3-chip we list the position, and the counts in the total $(0.5-7 \mathrm{keV})$, and the soft $(0.5-2 \mathrm{keV})$ band as well as the detection significance, $\sigma$. A conservative estimate for the error in the positions is 0.7 . In the second column we indicate - where appropriate - reasons to reject the source as the burster candidate: $\mathrm{o}$. position outside the WFC error circle, *. optical counterpart too bright, s. X-ray spectrum too hard (for discussion see text). In the second column we have also indicated the sources which are disturbed by a flickering pixel with $\mathrm{f}$.

\begin{tabular}{|c|c|c|c|c|c|c|}
\hline \# & note & $\begin{array}{c}\text { RA } \\
(\mathrm{J} 2000)\end{array}$ & $\begin{array}{c}\text { Dec } \\
(\mathrm{J} 2000)\end{array}$ & counts & soft & $\sigma$ \\
\hline \multicolumn{7}{|c|}{ SAX J1324.5-6313 } \\
\hline A & & $13^{\mathrm{h}} 24^{\mathrm{m}} 30.2^{\mathrm{s}}$ & $-63^{\circ} 12^{\prime} 41^{\prime \prime}$ & $5.9 \pm 2.4$ & $5.9 \pm 2.4$ & 3.1 \\
\hline B & $\mathrm{s}$ & $13^{\mathrm{h}} 24^{\mathrm{m}} 30.3^{\mathrm{s}}$ & $-63^{\circ} 13^{\prime} 50^{\prime \prime}$ & $77.9 \pm 8.9$ & $21.7 \pm 4.7$ & 33 \\
\hline $\mathrm{C}$ & & $13^{\mathrm{h}} 24^{\mathrm{m}} 38.0^{\mathrm{s}}$ & $-63^{\circ} 12^{\prime} 26^{\prime \prime}$ & $6.8 \pm 2.6$ & $6.8 \pm 2.6$ & 3.4 \\
\hline $\mathrm{D}$ & & $13^{\mathrm{h}} 24^{\mathrm{m}} 38.3^{\mathrm{s}}$ & $-63^{\circ} 13^{\prime} 28^{\prime \prime}$ & $19.5 \pm 4.5$ & $19.5 \pm 4.5$ & 9.2 \\
\hline $\mathrm{E}$ & & $13^{\mathrm{h}} 24^{\mathrm{m}} 39.4^{\mathrm{s}}$ & $-63^{\circ} 13^{\prime} 34^{\prime \prime}$ & $5.9 \pm 2.4$ & $5.9 \pm 2.4$ & 3.1 \\
\hline \multicolumn{7}{|c|}{ SAXJ1752.3-3128 } \\
\hline A & & $17^{\mathrm{h}} 52^{\mathrm{m}} 16.7^{\mathrm{s}}$ & $-31^{\circ} 39^{\prime} 46^{\prime \prime}$ & $10.7 \pm 3.3$ & $10.7 \pm 3.3$ & 5.3 \\
\hline B & & $17^{\mathrm{h}} 52^{\mathrm{m}} 30.6^{\mathrm{s}}$ & $-31^{\circ} 38^{\prime} 58^{\prime \prime}$ & $6.7 \pm 2.6$ & $4.8 \pm 2.2$ & 3.3 \\
\hline $\mathrm{C}$ & o & $17^{\mathrm{h}} 52^{\mathrm{m}} 39.4^{\mathrm{s}}$ & $-31^{\circ} 37^{\prime} 56^{\prime \prime}$ & $36.5 \pm 6.2$ & $35.9 \pm 6.1$ & 13 \\
\hline \multicolumn{7}{|c|}{ SAX J1806.5-2215 } \\
\hline A & o & $18^{\mathrm{h}} 06^{\mathrm{m}} 18.1^{\mathrm{s}}$ & $-22^{\circ} 15^{\prime} 39^{\prime \prime}$ & $13.7 \pm 3.9$ & $2.8 \pm 1.7$ & 5.7 \\
\hline B & o & $18^{\mathrm{h}} 06^{\mathrm{m}} 18.5^{\mathrm{s}}$ & $-22^{\circ} 17^{\prime} 24^{\prime \prime}$ & $48.4 \pm 7.1$ & $43.7 \pm 6.7$ & 19 \\
\hline $\mathrm{C}$ & o & $18^{\mathrm{h}} 06^{\mathrm{m}} 19.9^{\mathrm{s}}$ & $-22^{\circ} 18^{\prime} 03^{\prime \prime}$ & $7.9 \pm 2.8$ & $1.9 \pm 1.4$ & 4.1 \\
\hline D & & $18^{\mathrm{h}} 06^{\mathrm{m}} 31.7^{\mathrm{s}}$ & $-22^{\circ} 13^{\prime} 19^{\prime \prime}$ & $9.0 \pm 3.2$ & $5.5 \pm 2.4$ & 3.9 \\
\hline $\mathrm{E}$ & $*$ & $18^{\mathrm{h}} 06^{\mathrm{m}} 35.8^{\mathrm{s}}$ & $-22^{\circ} 15^{\prime} 01^{\prime \prime}$ & $10.5 \pm 3.3$ & $9.8 \pm 3.2$ & 5.0 \\
\hline $\mathrm{F}$ & $\mathrm{s}$ & $18^{\mathrm{h}} 06^{\mathrm{m}} 36.8^{\mathrm{s}}$ & $-22^{\circ} 15^{\prime} 26^{\prime \prime}$ & $14.9 \pm 3.9$ & $<0.66$ & 7.7 \\
\hline G & $\mathrm{f}$ & $18^{\mathrm{h}} 06^{\mathrm{m}} 37.4^{\mathrm{s}}$ & $-22^{\circ} 17^{\prime} 22^{\prime \prime}$ & $7.9 \pm 2.8$ & $6.9 \pm 2.6$ & 4.0 \\
\hline $\mathrm{H}$ & & $18^{\mathrm{h}} 06^{\mathrm{m}} 43.6^{\mathrm{s}}$ & $-22^{\circ} 16^{\prime} 06^{\prime \prime}$ & $8.7 \pm 3.0$ & $8.7 \pm 3.0$ & 4.3 \\
\hline I & $\mathrm{o}$ & $18^{\mathrm{h}} 06^{\mathrm{m}} 43.8^{\mathrm{s}}$ & $-22^{\circ} 18^{\prime} 42^{\prime \prime}$ & $13.8 \pm 3.7$ & $3.0 \pm 1.7$ & 6.9 \\
\hline \multicolumn{7}{|c|}{ SAXJ1818.7+1424 } \\
\hline A & o & $18^{\mathrm{h}} 18^{\mathrm{m}} 32.1^{\mathrm{s}}$ & $+14^{\circ} 22^{\prime} 09^{\prime \prime}$ & $45.3 \pm 6.9$ & $45.3 \pm 6.8$ & 21 \\
\hline B & o & $18^{\mathrm{h}} 18^{\mathrm{m}} 34.3^{\mathrm{s}}$ & $+14^{\circ} 26^{\prime} 29^{\prime \prime}$ & $6.8 \pm 2.6$ & $4.0 \pm 2.0$ & 3.5 \\
\hline $\mathrm{C}$ & $*$ & $18^{\mathrm{h}} 18^{\mathrm{m}} 35.6^{\mathrm{s}}$ & $+14^{\circ} 22^{\prime} 32^{\prime \prime}$ & $9.9 \pm 3.2$ & $9.9 \pm 3.2$ & 5.2 \\
\hline $\mathrm{D}$ & $*$ & $18^{\mathrm{h}} 18^{\mathrm{m}} 37.6^{\mathrm{s}}$ & $+14^{\circ} 22^{\prime} 44^{\prime \prime}$ & $36.6 \pm 6.1$ & $36.6 \pm 6.1$ & 18 \\
\hline $\mathrm{E}$ & & $18^{\mathrm{h}} 18^{\mathrm{m}} 37.8^{\mathrm{s}}$ & $+14^{\circ} 22^{\prime} 06^{\prime \prime}$ & $7.0 \pm 2.6$ & $6.0 \pm 2.4$ & 3.7 \\
\hline $\mathrm{F}$ & & $18^{\mathrm{h}} 18^{\mathrm{m}} 38.6^{\mathrm{s}}$ & $+14^{\circ} 22^{\prime} 59^{\prime \prime}$ & $27.8 \pm 5.3$ & $21.9 \pm 4.7$ & 14 \\
\hline G & & $18^{\mathrm{h}} 18^{\mathrm{m}} 48.3^{\mathrm{s}}$ & $+14^{\circ} 22^{\prime} 43^{\prime \prime}$ & $9.9 \pm 3.2$ & $9.9 \pm 3.2$ & 5.1 \\
\hline $\mathrm{H}$ & $\mathrm{o}$ & $18^{\mathrm{h}} 18^{\mathrm{m}} 55.8^{\mathrm{s}}$ & $+14^{\circ} 27^{\prime} 38^{\prime \prime}$ & $10.2 \pm 3.5$ & $7.8 \pm 3.0$ & 4.0 \\
\hline
\end{tabular}

stars found in the ROSAT All Sky Survey (Hünsch et al. 1999; here we use that for coronal sources with interstellar absorption columns up to $10^{21}$ atoms $\mathrm{cm}^{-2}$ the ROSAT/PSPC count rate is typically $1 / 3$ to $1 / 4$ of the Chandra count rate). Soft $\mathrm{X}$-ray transients with neutron stars have X-ray to optical flux ratios in quiescence several orders of magnitude higher than ordinary stars (e.g. Fig. 5 in Pooley et al. 2002a). Thus we conclude that the optically identified Chandra sources indicated in Table 3 are too bright in the optical for them to be the bursters.

Due to the small number of counts in each source, it is not possible to constrain the spectral shape of the X-ray emission. The most commonly used models to describe the quiescent emission of neutron star X-ray transients are $0.3 \mathrm{keV}$ black body radiation, $0.3 \mathrm{keV}$ Raymond-Smith emission, power-law emission with a slope of $\Gamma \sim 3$ or emission from a hydrogen
Table 4. USNO Digitized Sky Survey sources close to detected Chandra detections. For each source we give the position, $B$ and $R$ magnitudes, the distance $(\Delta)$ from the X-ray source, and the chance probability $P$ that the optical source is in the Chandra error-circle.

\begin{tabular}{ccccccc}
\hline \hline$\#$ & RA & Dec & $B$ & $R$ & $\Delta\left(^{\prime \prime}\right)$ & $P(\%)$ \\
\hline D & $13^{\mathrm{h}} 24^{\mathrm{m}} 38.284^{\mathrm{s}}$ & $-63^{\circ} 13^{\prime} 27.16^{\prime \prime}$ & 17.2 & 15.3 & 0.83 & 0.7 \\
\hline A & $17^{\mathrm{h}} 52^{\mathrm{m}} 16.723^{\mathrm{s}}$ & $-31^{\circ} 39^{\prime} 44.24^{\prime \prime}$ & 19.5 & 17.3 & 1.78 & 5.7 \\
$\mathrm{~B}$ & $17^{\mathrm{h}} 52^{\mathrm{m}} 30.537^{\mathrm{s}}$ & $-31^{\circ} 38^{\prime} 58.88^{\prime \prime}$ & 19.0 & 17.3 & 1.19 & 1.9 \\
\hline $\mathrm{D}$ & $18^{\mathrm{h}} 06^{\mathrm{m}} 31.589^{\mathrm{s}}$ & $-22^{\circ} 13^{\prime} 18.94^{\prime \prime}$ & 15.7 & 13.1 & 3.32 & 1.1 \\
$\mathrm{E}$ & $18^{\mathrm{h}} 06^{\mathrm{m}} 35.819^{\mathrm{s}}$ & $-22^{\circ} 15^{\prime} 00.87^{\prime \prime}$ & 16.1 & 14.8 & 0.28 & 0.0 \\
$\mathrm{H}$ & $18^{\mathrm{h}} 06^{\mathrm{m}} 43.734^{\mathrm{s}}$ & $-22^{\circ} 16^{\prime} 06.08^{\prime \prime}$ & 19.8 & 17.0 & 1.86 & 6.3 \\
\hline $\mathrm{C}$ & $18^{\mathrm{h}} 18^{\mathrm{m}} 35.593^{\mathrm{s}}$ & $+14^{\circ} 22^{\prime} 32.67^{\prime \prime}$ & - & 11.1 & 0.66 & 0.0 \\
$\mathrm{D}^{a}$ & $18^{\mathrm{h}} 18^{\mathrm{m}} 37.634^{\mathrm{s}}$ & $+14^{\circ} 22^{\prime} 44.44^{\prime \prime}$ & 8.6 & 7.4 & 0.0 & 0.0 \\
\hline
\end{tabular}

${ }^{a}$ Star HD 168344 with $V=7.6$.

atmosphere (e.g. Campana et al. 1998). Added to these models is a hard energy tail detected at high energies (e.g. Campana et al. 1998; Asai et al. 1996). Here we assume emission from a hydrogen atmosphere of a neutron star (Zavlin et al. 1996) plus a power-law, as was, for example, found for the quiescent emission of the neutron star X-ray transients Cen X-4 and Aql X-1 (Rutledge et al. 2001a, 2001b). We estimate the number of photons below $2 \mathrm{keV}$ using the average parameters found for Cen X-4 and Aql X-1, i.e. a power-law photon-index $\Gamma=1.0$, neutron star radius $R_{\infty}=16 \mathrm{~km}$, a neutron star temperature $k T=100 \mathrm{eV}$, and a ratio of the unabsorbed flux $(0.1-7 \mathrm{keV})$ expected from the $\mathrm{H}$-atmosphere to the power-law component of $5: 1$. For such a spectrum we compute the flux for a source with 1 Chandra count per second, and the ratio of counts at energies between 0.5 and $2 \mathrm{keV}$ to the total count rate. The resulting numbers are listed in Table 2. By comparison with the observed soft-to-total count ratios listed in Table 3 we can exclude sources which are harder than expected for soft X-ray transients in quiescence. We do not reject sources with soft spectra, because there is evidence that the photon-index of the power-law component could be higher than we have assumed (Campana et al. 1998). The two sources which are excluded in this way are indicated in Table 3 with s. Unacceptably high column densities are needed $\left(\simeq 10^{23}\right.$ atoms $\left.\mathrm{cm}^{-2}\right)$ to account for the lack of soft photons due to absorption (for source $\mathrm{F}$ of SAX J1806.5-2215 we do not detect anything below $2 \mathrm{keV}$ ). The fluxes do not significantly change if we assume the other spectral models or change the temperature and radius of the neutron star atmosphere model.

For all sources the distribution of the arrival times of the photons is compatible with a constant flux. Given the small number of photons for each source the limits on variability are not very constraining, but we can exclude that the flux measured is due to a flare lasting shorter than the exposure time.

Taking all these criteria into account we conclude that we have four candidate counterparts left for SAX J1324.5-6313 (source A, C, D and E), two for SAXJ1752.3-3128 (A and B), two for SAXJ1806.5-2215 (D and H), and three for SAX J1818.7+1424 (E, F and G). For SAX J1753.5-2349 we have no candidate. 

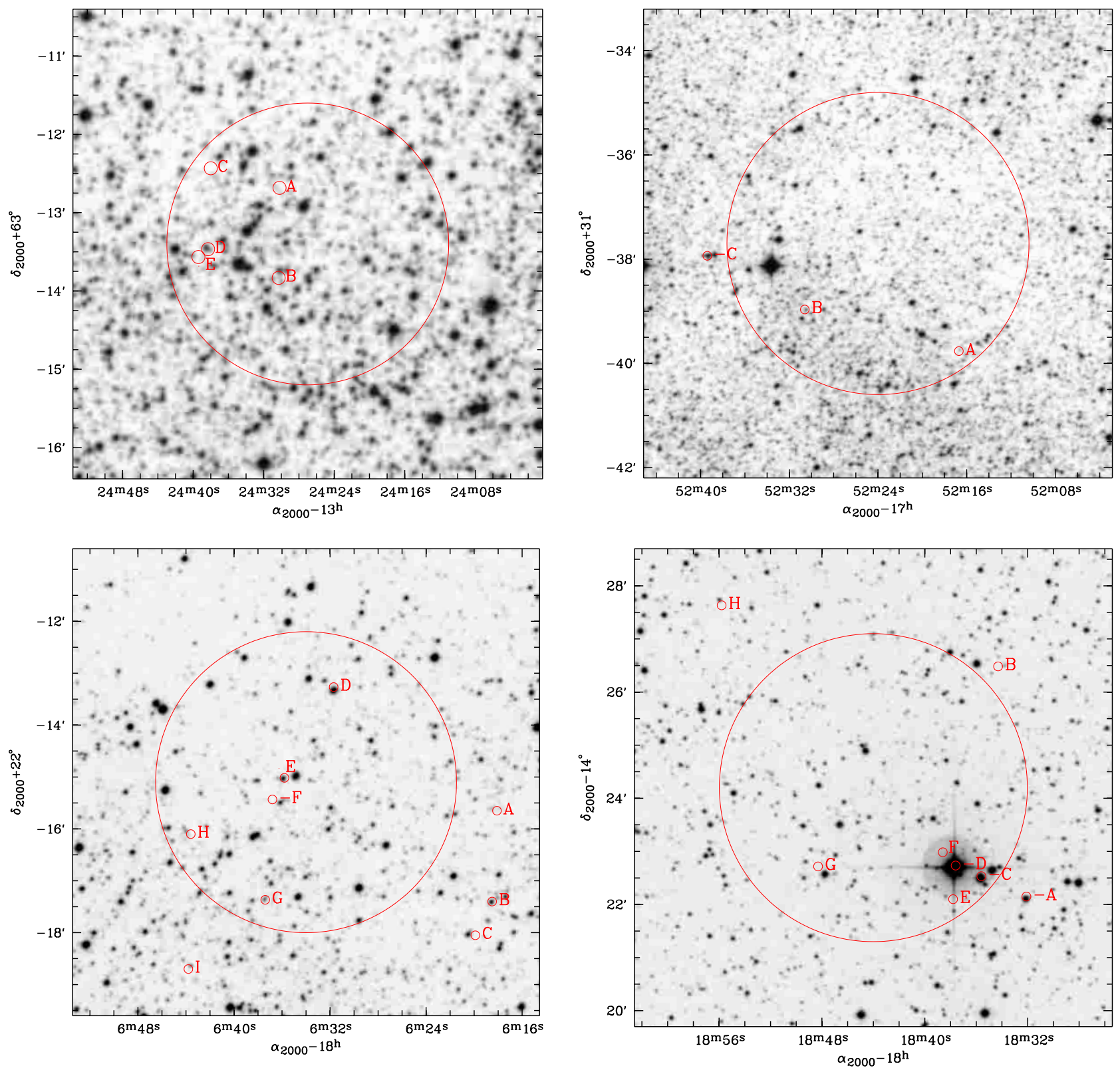

Fig. 1. Sources detected inside or close to the WFC error circles for SAXJ1324.5-6313, SAX J1752.3-3128, SAXJ1806.5-2215, and SAX J1818.7+1424, respectively. All detections are superposed on an image from the Digitized Sky Survey. The large circles indicate the WFC error circles. The error circles of all sources are increased to $5^{\prime \prime}$ for easier reference.

\section{SAX J1806.5-2215 and GRS 1741.9-2853}

In't Zand et al. (1998) reported the detection of two X-ray bursts from SAXJ1806.5-2215, and showed the analysis of the first burst. We take the opportunity of the present paper to report the detection of two additional type I bursts from SAX J1806.5-2215 during WFC observations on MJD 50537.91 and MJD 50732.90. No persistent emission is observed for this source in any WFC observation. The first and strongest burst (on MJD 50325.88) has a duration of $\simeq 150 \mathrm{~s}$, all other bursts last $\simeq 20 \mathrm{~s}$. The observed spectra can be well described by an absorbed black body model with temperatures between 1.7 and $2.2 \mathrm{keV}$. The unabsorbed bolometric peak flux of the strongest burst is $(2.6 \pm 1.2) \times 10^{-8} \mathrm{erg} \mathrm{cm}^{-2} \mathrm{~s}^{-1}$.
This gives an upper-limit on the distance of $8.0 \mathrm{kpc}$ and neutron star radii between 4.8 and $7.0 \mathrm{~km}$, assuming that the peak flux is below the Eddington limit of $L_{\mathrm{Edd}}=2 \times 10^{38} \mathrm{erg} \mathrm{s}^{-1}$. The waiting times between the four bursts are 41, 171 and 195 days, respectively.

Recently the ASM lightcurve of SAX J1806.5-2215 became available. It shows a faint but clear detection between March 1996 and October 1997. This coincides with the same period as the occurence of the four X-ray bursts observed with the WFC. The maximum persistent flux was $\simeq 2 \times$ $10^{-10} \mathrm{erg} \mathrm{cm}^{-2} \mathrm{~s}^{-1}(2-10 \mathrm{keV})$, and slowly decreased over time. This is comparable to the upper-limit derived during the WFC observations. Assuming the distance derived above, this corresponds to a luminosity of $\simeq 2 \times 10^{36} \mathrm{erg} \mathrm{s}^{-1}$. 
GRS 1741.9-2853 was in the field of view of a $47.2 \mathrm{ks}$ ROSAT/PSPC pointed observation of the Galactic center region obtained on March 2-9, 1992. It is not detected, and we determine an upper-limit of $0.0003 \mathrm{cts} \mathrm{s}^{-1}$ (channels 50240 ), corresponding to an unabsorbed luminosity in the 0.5 to $2.5 \mathrm{keV}$ range of $3 \times 10^{34} \mathrm{erg} \mathrm{s}^{-1}$ at the distance of $7.2 \mathrm{kpc}$, for an assumed power-law spectrum with photon index $1 \mathrm{ab}-$ sorbed by a column $N_{\mathrm{H}}=10^{23}$ atoms $\mathrm{cm}^{-2}$ (see Cocchi et al. 1999). This proves that GRS $1741.9-2853$ is a burster with low persistent emission.

\section{Discussion}

Three of the nine burst sources with low persistent emission discovered with the WFC were observed during ROSAT observations at luminosities of $\simeq 10^{34-35} \mathrm{erg} \mathrm{s}^{-1}$ a few years prior to the X-ray burst (Kaptein et al. 2000; Cornelisse et al. 2002; this paper). If the five burst sources of Table 2 had similar luminosities and spectra, their countrate with Chandra would be several orders of magnitude higher than the countrates of the sources listed in Table 3. Instead, the luminosities of the burst sources are at $\simeq 10^{33} \mathrm{erg} \mathrm{s}^{-1}$, comparable to the BeppoSAX/NFI observations of SAX J2224.9+5421 (Antonelli et al. 1999; in 't Zand 2002 , in preparation) and in the range of quiescent soft X-ray transients with neutron stars (e.g. Campana et al. 1998).

With the interstellar hydrogen column and upper limits to the distances listed in Table 2 and the spectrum described in Sect. 3, we compute the unabsorbed flux and upper limits to the luminosities between 0.5 and $7.0 \mathrm{keV}$. For the brightest candidate counterparts of SAX J1324.5-6313, SAX J1752.3-3128, SAXJ1806.5-2215 and SAXJ1818.7+1424 we obtain upper limits to the unabsorbed persistent luminosity of $4 \times 10^{32}, 3 \times 10^{32}, 2 \times 10^{32}$ and $4 \times 10^{32} \mathrm{erg} \mathrm{s}^{-1}$, respectively. From the upper-limit derived from the observation of SAX J1753.5-2349 we get a luminosity of $<4 \times 10^{32} \mathrm{erg} \mathrm{s}^{-1}$ $(0.5-7 \mathrm{keV})$. These luminosities are indeed in the range expected for quiescent soft $\mathrm{X}$-transients with a neutron star.

Because our Chandra observations give more than one possible counterpart, several possible counterparts must be chance coincidences. This is in agreement with known $\log N-\log S$ distributions, which predict $\simeq 5$ sources in the field of view (see e.g. Rosati et al. 2002). This raises the question whether all Chandra sources are chance coincidences, i.e. whether we have not detected the actual counterparts for the bursters. Given that these systems are neutron star low mass X-ray binaries, we compare them to known other systems, i.e. the soft X-ray transients in quiescence. The lowest X-ray luminosities detected for quiescent soft X-ray transients are $\sim 10^{32} \mathrm{erg} \mathrm{s}^{-1}$ (e.g. Cen X-4, Campana et al. 1998). This is around the detection limit for the Chandra observations discussed in this paper. We therefore consider it possible that we actually have detected the persistent flux of the bursters, and that they are soft X-ray transients in quiescence, for which no outburst has as yet been detected. If so, this implies that their actual distances are not much less than the upper limits listed in Table 2.

The persistent luminosities of the bursters observed with Chandra is well below the limit set with the WFC observations. This means that we cannot exclude that the persistent luminosity during the WFC observations was $\sim 10-100$ times higher than detected with Chandra, and that it was this higher flux level which triggered the burst. The detections with ROSAT of 1RXS J171824.2-402934 and SAX J1828.5-1037, and of GRS 1741.9-2853 with GRANAT combined with nondetections at other epochs, show that the persistent flux level of these sources is variable.

The energy released during a burst due to nuclear fusion is about $1 \%$ of the accretion energy of the matter accreted onto the neutron star (see e.g. Lewin et al. 1993). Dividing the fluence of the bursts detected with the WFC by $1 \%$ of the persistent emission detected by Chandra we estimate burst intervals of $\sim 10$ years. If only $1 / 6$ th of the persistent flux is due to accretion, the remainder being due to the cooling of the neutron star (i.e. if only the power-law component is due to accretion, see Sect. 3) the estimated burst intervals rise to $\sim 60$ years. It is also suggested that the power-law component during quiescence is not due to accretion (see e.g. Campana et al. 1998), and this means that the waiting time derived above is an under-limit. This explains why these events are so rare, and why we have only seen one burst for most of these sources.

This raises the question how many of these burst sources with low persistent emission exist in our Galaxy. With the WFC the Galactic Center region is observed every half year since 1996, for a total observation time of $5.5 \times 10^{6} \mathrm{~s}$ up to end 2001. If we assume the Galactic distribution of low-mass X-ray binaries derived by van Paradijs \& White (1995), $\simeq 50 \%$ of the population is in the field of view $\left(40^{\circ} \times 40^{\circ}\right)$ of the WFC. During all Galactic center observations 5 bursters with low persistent emission have been detected, i.e. SAX J1752.3-3128, SAX J1753.5-2349, SAX J1806.5-2215, 1RXS J171824.2-402934, and GRS 1741.9-2853 (the other four are outside the Galactic center region). This gives an average waiting time between the detection of these bursters of $1.1 \times 10^{6} \mathrm{~s}$. If we also assume that the waiting time between burst of one source is 60 years $\left(1.9 \times 10^{9} \mathrm{~s}\right)$ we expect $\simeq 2 \times 10^{3}$ sources in the Galactic center region, giving $4 \times 10^{3}$ sources in the whole Galaxy. If on the other hand these sources are extensive periods of time at a persistent luminosity of $10^{34} \mathrm{erg} \mathrm{s}^{-1}$, as the detections of SAX J1828.5-1037, 1RXS J171824,2-402934, and GRS 1741.9-2853 suggests, the waiting time drops to 0.5 year (see Table 1). This gives a number of 30 sources in our Galaxy. We conclude that the estimates for the total number of X-ray bursters with low persistent fluxes range from 0.5 to 60 times the number of known bursters $(\simeq 70)$.

In this respect it is interesting to note that the first Chandra observations of globular clusters indicate that these systems harbour more quiescent soft X-ray transients than bursters with high persistent fluxes. For example, Liller 1, NGC 6440 and NGC 6652 all contain such quiescent sources in addition to the bright source (Homer et al. 2001; Pooley et al. 2002b; Heinke et al. 2001); and 47 Tuc, $\omega$ Cen, NGC 6752 and NGC 6397 contain quiescent sources but no bright source (Grindlay et al. 2001a, 2001b; Rutledge et al. 2001c; Pooley et al. 2002a). The formation mechanism for low-mass X-ray binaries in globular clusters (tidal capture or exchange encounter; see review by Hut et al. 1992) is different from the formation mechanism in 
the galactic disk (evolution of a primordial binary). If the ratio of quiescent to bright X-ray bursters depends on the formation mechanism, we do not necessarily expect comparable ratios in the cluster and in the Galactic disk.

If bursts can arise from quiescent systems, we must consider the possibility that a burst from a globular cluster is due to a dim source, rather than to the bright source in it. This would undermine the argument that a burst from a cluster proves that the bright source in it is a neutron star. Nonetheless, we think that the argument holds in all eleven cases where it has been applied so far, as bursts from dim sources are extremely rare. For example, we have detected $\sim 2200 \mathrm{X}$-ray bursts in our WFC observations of the Galactic center region; only five of these are from dim sources. Indeed, bursts from the globular cluster NGC 6440 were detected only when the transient in this cluster was active (in 't Zand et al. 2001).

\section{References}

Antonelli, L. A., Gandolfi, G., \& Feroci, M. 1999, GCN notice, 445

Bildsten, L. 1998, in The many faces of neutron stars, ed. J. Bucheri, J. van Paradijs, \& M. A. Alpar (Kluwer, Dordrecht), 419

Bildsten, L. 2000, in Cosmic explosions, ed. S. S. Holt, \& W. W. Zhang, AIP, E65

Campana, S., Colpi, M., Mereghetti, S., Stella, L., \& Tavani, M. 1998, A\&AR, 8, 279

Cocchi, M., Bazzano, A., Natalucci, L., et al. 1999, A\&A, 346, L45

Cocchi, M., Bazzano, A., Natalucci, L., et al. 2001, A\&A, 378, L37

Cornelisse, R., Verbunt, F., in 't Zand, J. J. M., et al. 2002, A\&A, 392, 885

Dickey, J. M., \& Lockman, F. J. 1990, ARA\&A, 28, 215

Freeman, P. E., Kashyap, V., Rosner, R., \& Lamb, D. Q. 2002, ApJS, 138, 185

Fujimoto, M. Y, Sztajno, M., Lewin, W. H. G., \& van Paradijs, J. 1987, ApJ, 319, 902
Grindlay, J., Heinke, C., Edmonds, P., \& Murray, S. S. 2001a, Science, 292, 2290

Grindlay, J., Heinke, C., Edmonds, P., Murray, S. S., \& Cool, A. M. 2001b, ApJ, 563, L53

Heinke, C., Edmonds, P., \& Grindlay, J. 2001, ApJ, 562, 363

Homer, L., Deutsch, E. W., Anderson, S. F., \& Margon, B. 2001, AJ, 122,2627

Hünsch, M., Schmitt, J. H. M. M., Sterzik, M. F., \& Voges, W. 1999, A\&AS, 135, 319

Hut, P., McMillan, S., Goodman, J., et al. 1992, PASP, 104, 981

in 't Zand, J. J. M., Heise, J., Muller, J. M., et al. 1998, Nucl. Phys. B, 69/1-3, 228

in 't Zand, J. J. M., van Kerkwijk, M. H., Pooley, D., et al. 2001, ApJ, 563, L41

Kaptein, R. G., in 't Zand, J. J. M., Kuulkers, E., et al. 2000, A\&A, 358, L71

Koyama, K., Inoue, H., \& Makishima, K. 1981, ApJ, 247, L27

Lewin, W., van Paradijs, J., \& Taam, R. 1993, Space Sci. Rev., 62, 223

Matsuoka, M., Inoue, H., Koyama, K., et al. 1980, ApJ, 240, L137

Pavlinsky, M. N., Grebenev, S. A., \& Sunyaev, R. A. 1994, ApJ, 425, 110

Pooley, D., Lewin, W., Homer, L., et al. 2002a, ApJ, 569, 405

Pooley, D., Lewin, W., Verbunt, F., et al. 2002b, ApJ, submitted [astro-ph/0111212]

Rosati, P., Tozzi, P., Giacconi, R., et al. 2002, ApJ, 566, 667

Rutledge, R. E., Bildsten, L., Brown, E. F., Pavlov, G. G., \& Zavlin, V. E. 2001a, ApJ, 551, 921

Rutledge, R. E., Bildsten, L., Brown, E. F., Pavlov, G. G., Zavlin, \& V. E. 2001b, ApJ, 559, 1054

Rutledge, R. E., Bildsten, L., Brown, E., et al. 2001c, ApJL, submitted [astro-ph/0105405]

van Paradijs, J., \& White, N. 1995, ApJ, 447, 33L

Weisskopf, M. C. 1988, Space Sci. Rev., 47, 47

White, N. E., Kaluzienski, J. L., \& Swank, J. H. 1984, in High energy transients in astrophysics, ed. S. E. Woosley, AIP, 31

Zavlin, V. E., Pavlov, G. G., \& Shibanov, Y. A. 1996, A\&A, 315, 141 